\title{
The Research and Application of MRF Magnetic Circuit Design
}

\author{
Yuan Shu*, Ren Hongjuan and Luo Yiping \\ Faculty of Automotive Engineering, Shanghai University of Engineering and Science, Shanghai, 201620, P.R. China
}

\begin{abstract}
In this paper, the lord magnetic field of MRF and its circuit design are mainly expounded, according to the loading ways of magnetic field and the magnetic circuit design plans of magneto-rheological fluid devices at present, the general form and characteristics are summarized. In addition, a kind of magneto-rheological fluid dynamometer is proposed in this paper, in terms of its magnetic circuit structure, a magnetic-field-lord test bench about magnetorheological fluid is designed; a preliminary theoretical research is also given in order to improve the performance of magneto-rheological fluid dynamometer.
\end{abstract}

Keywords: MRF, magnetic circuit design, magnetizing coil.

\section{INTRODUCTION}

As a new intelligent material, magneto-rheological fluid can change itself into so called "similar solid" instantaneously as long as a magnetic field is applied on it, consequently, the magnetic field is a key factor to ensure the normal work of magneto-rheological fluid and the research of magnetic field which is applied on MRF is particularly important. In general, under the condition of the normal work of magneto-rheological fluid, the magnetic field surrounding the MRF should be distributed as uniform as possible, in order to guarantee the stability of MRF during working time. Besides, the magnetic field should cover all the MRF participate in the work, the condition that some magneto-rheological fluid have no magnetic field applied on is not allowed. The choices of material and working point of magnetic circuit design, as well as magnetic flux leakage and energy loss are the key content that should take into consideration. For the reason that the researches of magnetic field and its circuit design play a pivotal role in MRF development, and the important research value of MRF itself, according to the domestic and foreign research results on magnetic circuit of magneto-rheological fluid devices, this paper mainly carries on the induction analysis for MRF magnetic field and its circuit.

\section{THE MAGNETIC PROPERTIES OF MRF}

For MRF, each of the ferromagnetic particles can be seen as a small magnet. Among these magnets, there is a cross coupling effect between each adjacent atom, it is so called magnetic domain which can prompt adjacent atomic magnetic moment arranged in parallel, and then a magnetizing saturated area formed.

While without the effect of magnetic field, the direction of every atomic magnetic moment is same for each magnetic domain, but the different magnetic domain has different magnetic moment direction. The arrangement of magnetic domain makes the effect between each magnetic domain cancellation, and every particle in a steady state with minimum energy. Therefore, all of the particles' average magnetic moment is zero and without any magnetic.

While with the effect of magnetic field, because of the magnetic energy the magnetic moments hold, which have same direction with the field, is lower than those who have different direction with the magnetic field, the angle of magnetic moment is big and the volume of magnetic domain decreases gradually. At this moment, the particles' average magnetic moment is not zero and shows magnetic, and then the particles connect into a sequence chain.

When external magnetic field strength is very small, for isotropic media, at any point of the magnetic medium, the magnetic field strength is proportional to the magnetization:

$\mathrm{M}_{\mathrm{s}}=\chi_{0} \mathrm{H}$

where, $M_{s}$ is magnetization; $\chi_{0}$ is magnetic susceptibility, which is a dimensionless number; $H$ is magnetic field strength.

With increasing of the magnetic field strength:

$\mathrm{M}_{\mathrm{s}}=\mathrm{B} / \mu_{0}-\mathrm{H}$

where, $B$ is magnetic induction intensity, $\mu_{0}$ is permeability of vacuum;

and:

$\mu=\mathrm{B} / \mathrm{H}$

$\mu_{\mathrm{r}}=\mu / \mu_{0}$

then:

$\mathrm{M}_{\mathrm{s}}=\left(\mu_{\mathrm{r}}-1\right) \mathrm{H}$

where, $\mu$ is permeability; $\mu_{r}$ is relative permeability. With the further increase of magnetic field intensity, MRF reaches full saturation. 


\section{THE STATUS OF MRF MAGNETIC CIRCUIT DESIGN}

Apparently, the study of MRF is in a stage of rapid rise, thegood or bad of MRF magnetic circuit design has a direct impact on MRF device working performance.

\subsection{The MRF Magnetic Circuit Design of MRF Shock Absorber}

The working principle of magneto-rheological fluidshock absorber is using controller to control the current of shock absorber in order to change theinternal magnetic field strength, so that the viscosity of MRF in the damping channel may be changed and shock absorber's damping changes also. Lord Company in the USA has developeda kind of Magneto-rheological shock absorber seat for trucks in the early 90's, and University of Nevada developed a magneto-rheological fluid shock absorber for "Hummer" military jeeps which can make jeeps' cross-country speed increased $30 \%-40 \%$ [1]; In the domestic, Chongqing Institute of Opto Electronics, Harbin Institute of Technology put more and more resources into the research of MRF magnetic circuit design [2]. There are three operating modes of Magneto-rheological fluid: flow, shear and extrusion, for magneto-rheological fluid shock absorber, shear mode and mixed mode are used mainly. Normally, a magnetorheological fluid shock absorber can be composed of a piston, piston flank, damping channel, magnetism conducting cylinder, they form a closed loop, as shown in Fig. (1), among them, the piston part should consist of electromagnetic coils, damping channel containing magnetorheological fluid. Electromagnetic coil generates a magnetic field, magnetic lines of force by the piston through the pistonflank (magnetic yoke), and then through damping channel gap, magnetism conducting cylinder, another part of the damping channel, magnetic yoke in turn, finally through piston structure to form a closed loop.

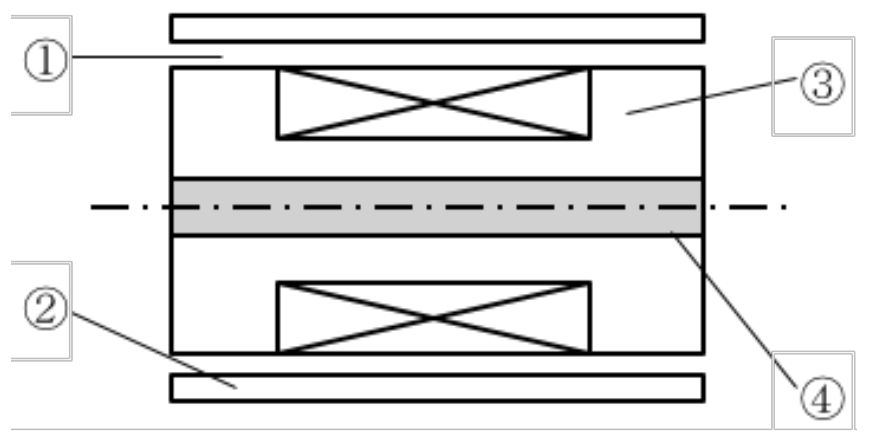

(1) damping channel(2)magnetism conducting cylinder(3)piston flank(4)piston

Fig. (1). The magnetic circuit structure of MRF shock absorber.

\subsection{The MRF Magnetic Circuit Design of MRF Clutch}

The MRF clutch is a new type of speed regulating device which can transmit the power by using MR effect [3]. The structures of the magneto-rheological fluid clutch are usually cylinder type,d isk type and cylindrical type, the most commonly used for cylinder type and disk type, whichmainly rely on the shear yield stress of MRF to transmit motion and power.

The working principle of MRF clutch can be simply described as: there is full magneto-rheological fluid between the active and passive parts of the clutch, while with the effect of magnetic field, magneto-rheological fluid change itself into so called "similar solid"instantaneously, some magnetic chainsare formedbetween the active and passive parts, the chains will be cut as long as there is a speed difference between the two parts, so shear yield stress is produced and the active part will drive the passive part through MRF. As long as there is sufficient shear stress generated, enough torque can be transferred, so that MRF clutch can make the active and passive parts rotate synchronously, that is the function of clutch called "combined with".

\subsubsection{The Cylindrical Magneto-Rheological Fluid Clutch}

The cylindrical magneto-rheological fluid clutch is mainly composed of outer cylinder, inner cylinder, active pumping and driven shaft, as shown in Fig. (2): inner cylinder comprises electromagnetic coils, driving shaft is connected with inner cylinder, and driven shaft is connected with outer cylinder. In Fig. (2), dotted lines for the magnetic field lines, they come from inner cylinder in turn through MRF channel (working gap), outer cylinder, another part of the MRF channel, and go into inner cylinder finally to form a closed loop. Chen Xu etc. form Chongqing University of Science and Technology carried out a finite element analysis of magnetic field of cylindrical magneto-rheological clutch [4], the result showed that most magnetic field lines produced by coils passed vertical through the working gap, besides, all of them were in the inner part of the clutch; the values of theoretical calculation were compared with the finite element calculation values, it proved the correctness of finite element model.

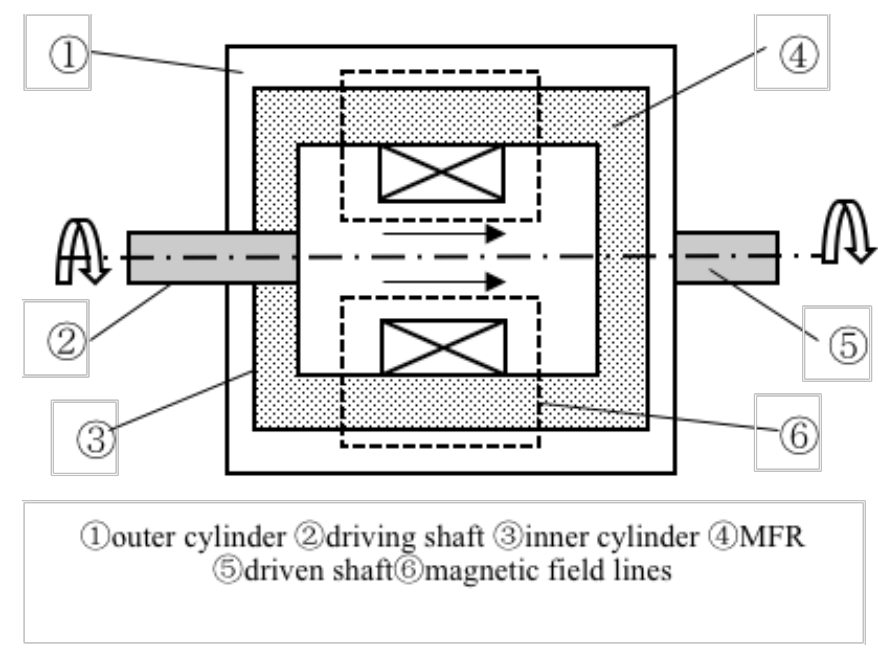

Fig. (2). The magnetic circuit structure of cylindrical MRF clutch.

\subsubsection{The Disk Magneto-Rheological Fluid Clutch}

Generally, there are two types of MRF clutch: double disks and multi disks. Fig. (3) is the magnetic circuit structure of disk MRF clutch, and there is a MRF channel between driving disk and driven disk, the magnetic field lines produced by coils pass through shell, driving disk, 
working gap, driven disk and back to shell finally to form a closed loop. Compared with cylindrical MRFclutch, the mechanical structure of disk MRF clutch is more simple and compact; therefore, the design of magnetic circuit is easier.

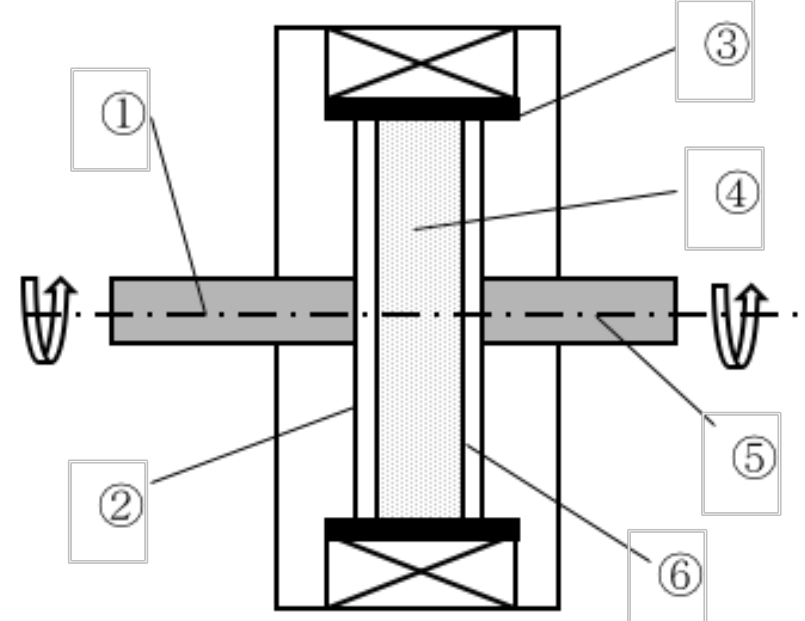

(1)driving shaft(2)driving disk(3)oil seal(4)MRF (5)driven shaft(6) driven disk

Fig. (3). The magnetic circuit structure of cdisk MRF clutch.

\subsection{The Design of Magnetizing Coils}

Taking all MRF devices available into consideration, because of a series of limiting factors just as mechanical construction requirements, mechanical processing level and so on, the type of magnetizing coils of MRF devices is single group often, shown in Fig. (4). But from the perspective of magnetic field design, the design of magnetic circuit should have an optimization function on devices. Zheng Lin et al. from Chongqing University gave another two coil winding methods on the basis of single group [5], as shown in Figs. $(5,6)$. After the theoretical calculation and finite element analysis, they found out that the double groups with different winding directions can greatly improve the flux density of working gap, in addition, it also has faster response speed.

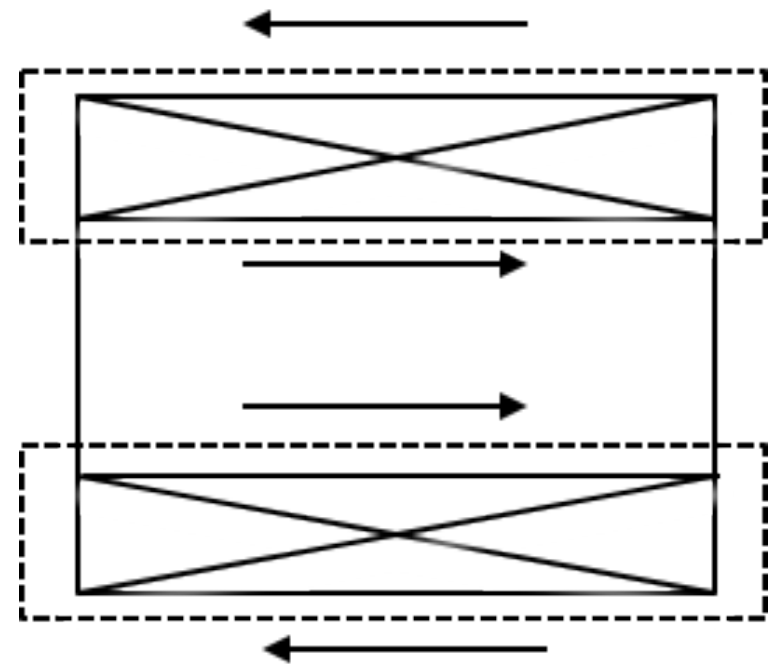

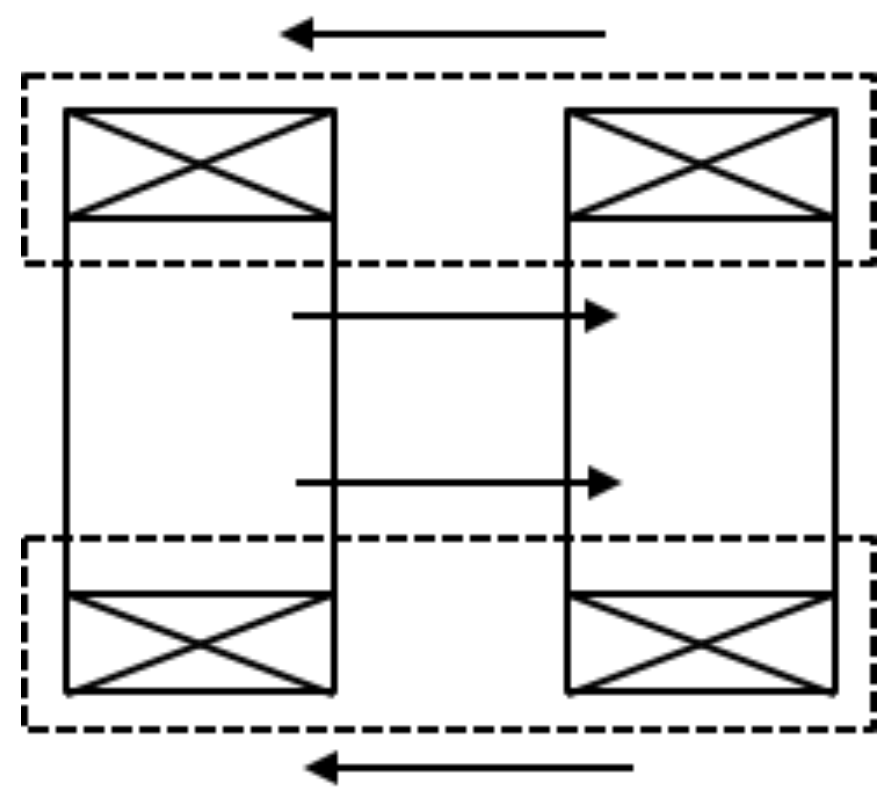

Fig. (5). The coils of double group with same winding direction.
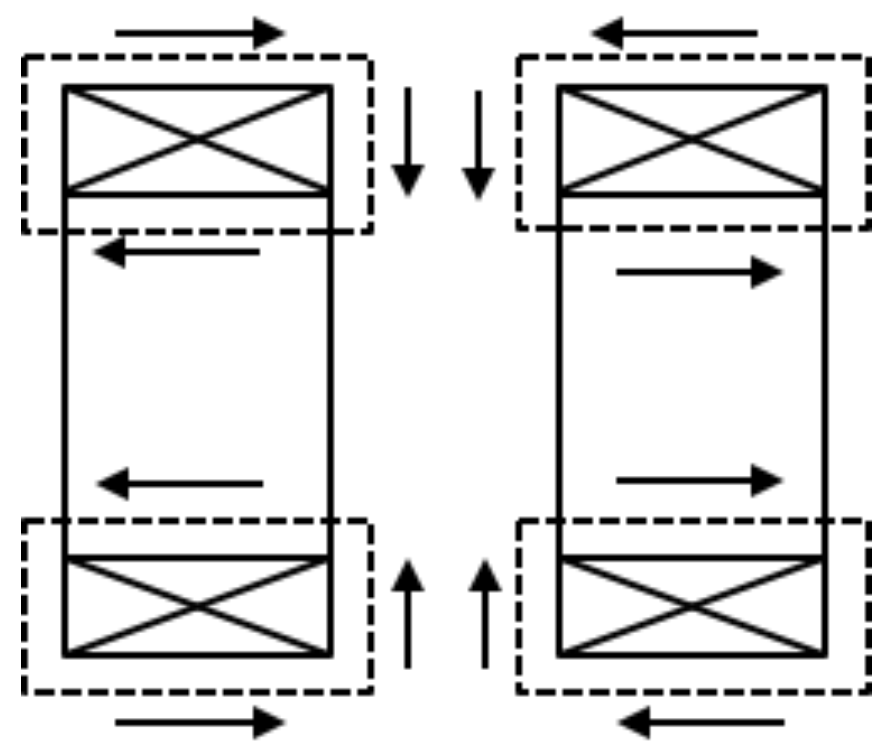

Fig. (6). The coils of double group with different winding directions.

In addition to the above three kinds of excitation coil winding ways, according to the structure characteristics of MRF devices, changing the shape of the yoke is also one of the effective methods of magnetic circuit design, for instance, bracelet ring coils and straight-flanked ring coils [6], as shown in Figs. $(\mathbf{7}, \mathbf{8})$. In these pictures, dotted lines for the magnetic field lines, according to the related theories, compared with the whole ring, the MRF channel is small enough, therefore the magnetic field lines passage through the MRF can be regarded as uniform distribution and equip with a high magnetic flux density, what will ensure the high efficiency of MRF.

Fig. (4). The coils of single group. 


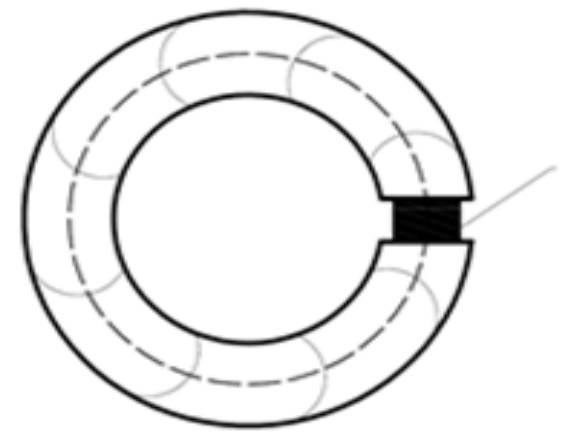

MRF channel

Fig. (7). Bracelet Ring Coils.

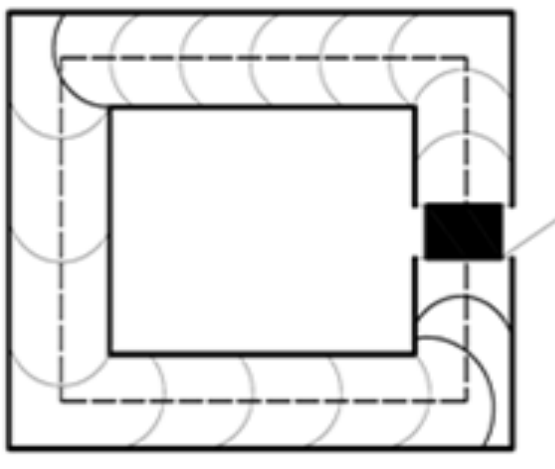

\section{MRF channel}

Fig. (8). Straight-flanked Ring Coils.

\section{THERESEARCHPROSPECT OF MRF MAGNETIC FIELD}

MRF engine dynamometer is a new type of power measuring device which combines MRF with dynamometer. Dynamometer offers load for the output power of the test machine, takes in mechanical energy output by the test machine, simultaneously the dynamic performance parameters of the test machine such as torque and rotational speed and so on is measured. Dynamometer is based on the principle that action and reaction are equal and opposite direction to measure torque. MRF engine dynamometer relies mainly on the shear stress of MRF to transmit torque. MRF engine dynamometer is a kind of MRF transmission devices, nowadays, MRF transmission is still in the early stage of development, if successfully developed, it is of great significance. And the reasonable design of magnetic circuit is one of the most important factors to determine the success or failure of MRF engine dynamometer development. This paper presents a MRF dynamometer which is in the research and development stage, as shown in Fig. (9) is its magnetic circuit.

This magneto-rheological fluid dynamometer consists of active plate and drive shaftonly, there is no driven part, so it is a MRF transmission device which just with a single disk, the MRF channel is between the active plate and the right shell.

Due to the structure of MRF dynamometer is relatively complex, it is not easy to magnetic field analysis. By simplifying the existing structure of MRF dynamometer, removing the rotating parts of magneto-rheological fluid dynamometer, keeping the MRF channel only, this paper presents a magnetic-field-lordtest bench about magnetorheological fluid, as shown in Fig. (10). This text bench is just used to research the influence of lording magnetic field on MRF, the magnetic field characteristics of magnetic rheological liquid working mode is not taken into consideration.

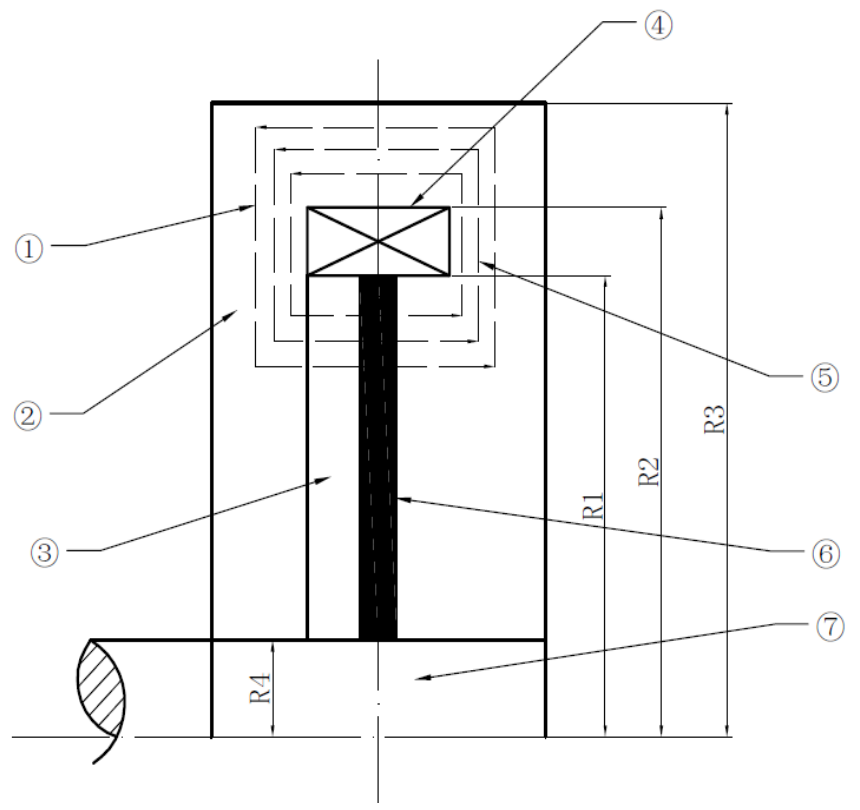

(1) outer ring (2)left shell (3)rotary table (4)coils (5)right shell (6) MRF (7) rotary shaft

Fig. (9). The magnetic circuit structure of MRF dynamometer.

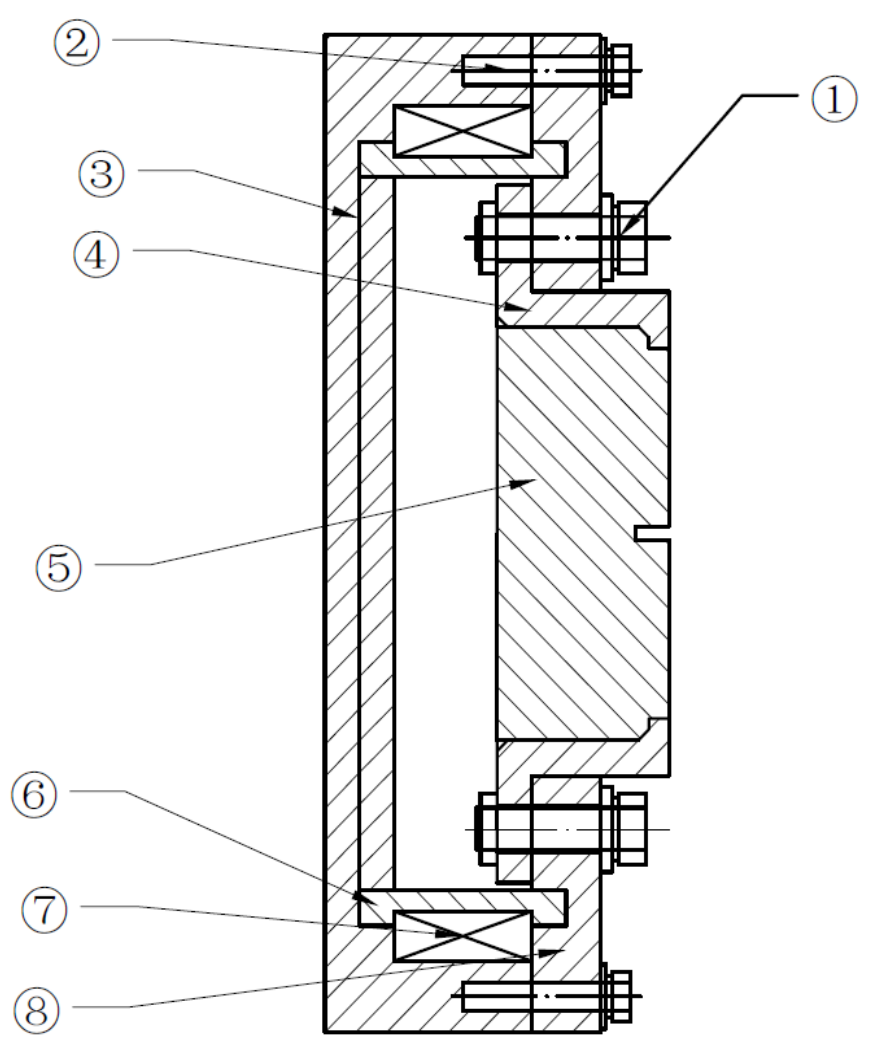

(1), (2)bolts (3)left shell(4)outer inductive block(5)inner inductive block(6)magnetic yoke(7)coils(8)right end cover

Fig. (10). The mechanical structure of MRF magnetic-field-lord test bench. 
Considering the influence of the number of turns, the winding method of coils on MRF, the text bench this paper presented is a detachable structure, so it is convenient to adjust the coils at any time. In Fig. (10), the material of inner inductive block is DT4C electromagnetic iron, it contact with MRF, when there is a magnetic field applied, a high precision Tesla meter is used to measure the magnetic field power of the inner inductive block on some points, according to some related theories, the power of MRF magnetic field can be obtained indirectly, so it can provide a data reserve for MRF magnetic circuit design, structural optimization and so on.

\section{CONCLUSION}

(1) According to the characteristics of MRF, a kind of magneto-rheological fluid dynamometer is proposed in this paper, this is a "single disk" MRF transmission device, which hasn't been seen in the current research results. Although a more detailed and specific instruction about this MRF engine dynamometer is not given, the further research of it will continue and more information will be put forward in the near future.

(2) On the basis of this dynamometer, a magnetic-fieldlordtest bench about magneto-rheological fluid is presented in this paper, the mechanical structure of this test bench is proposed. The key point is to study the effect of different loading modes of magnetic field on magnetic rheological fluid field, but the text is still in progress and the test results are need to be verified.

\section{ABOUT THE AUTHORS}

First Author Yuan Shu, graduate students of Shanghai University of Engineering and Science. The author graduated from Nanjing Forestry University with a bachelor degree.
The author's major is automotive engineering and 1 paper and 1 patent had been published.

Second Author Ren Hongjuan, University associate professor of Shanghai University of Engineering and Science.

Third Author Luo Yiping is professor level senior engineer of Shanghai University of Engineering and Science.

\section{CONFLICT OF INTEREST}

The author confirms that this article content has no conflict of interest.

\section{ACKNOWLEDGEMENTS}

This research was supported by the construction of professional platform for modern automobile service engineering in Shanghai city (XKCZ1214).

\section{REFERENCES}

[1] F. Gordaninejad, and S.P. Kelso, "Magneto-rheological fluid shock absorbers for hmmwv," In: Proceedings of SPIE Conference on Smart Structures and Materials on Damping and Isolation, vol.3989, New port Beach, CA,March, 2000, pp. 3989-21.

[2] Z. Hongjun, L. Changrong, T. Xudong, "Magnetic field finite element application in estimation performance of magnetorheological damper," Journal of Chongqing University, vol.28, no. 10, pp. 9-12, 2005.

[3] W. Jianxiao, and M. Guang. "MRF device and its application in mechanical engineering," Mechanical Strength, vol. 23, no. 1, pp. $50-56,2001$

[4] C. Xu, and L. Lijun. "The finite element analysis of cylindrical magneto-rheological fluid clutch's magnetic field," Mining Machinery, vol. 42, no. 2, pp. 24-28, 2014.

[5] Z. Lin, L. Yinong, H. Yong, and T. Xu. "The magnetic circuit design and structure optimization of magneto-rheological damper," Journal of Vibration Engineering, vol. 21, no. 2, pp. 173-178, 2008.

[6] Y. Zhao, "Engineering Electromagnetic Field," Xi'an:Xi'an Jiao Tong University press, 2013. 